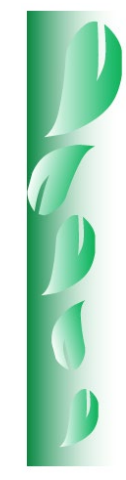

DOI: https://doi.org/10.29298/rmcf.v12i66.819

\title{
Morfotipos de ectomicorrizas en una cronosecuencia de rodales de Pinus patula Schiede ex Schltdl. \& Cham. en la zona este de México
}

\section{Ectomycorrhizae morphotypes in a Pinus patula Schiede ex Schltdl. \& Cham. stand chronosequence in eastern Mexico}

\author{
Yajaira Baeza Guzmán ${ }^{1}$, Jesús Dorantes López ${ }^{1}$, Rosario Medel Ortiz ${ }^{2}$ \\ y Dora Trejo Aguilar ${ }^{1 *}$
}

\begin{abstract}
Forest activity for timber purposes in Mexico is based on the harvesting of various pine species, one of the mostly used is Pinus patula. Ectomycorrhizal (ECM) fungi increase seedling survival, and, therefore, their use ensures the success of forest plantations. Reforestation with native pine species increases the diversity of ectomycorrhizal fungi. The aim of this work was to compare species diversity associated with stand age and to determine the changes in the fungal community at the morphotype level. The study was carried out in a commercial plantation, with stands aged 2, 5, 7, 9, 14 to 25 and > 50 years. Mycorrhizal roots were collected at the beginning of the rainy season and grouped according to their morphology, and the diversity index, percentage of abundance and relative frequency were determined. Twenty-eight morphotypes were determined, including two of the Laccaria genus, two of Tomentella and one of Cortinarius. There is a positive correspondence between the number of morphotypes and the age of the pines. Mature stands (14-25 and >50 yr-old) were more diverse than young ( 2 to $9 \mathrm{yr}$-old) stands, with the exception of the 7 year-old stand, which showed similar diversity values to the mature stands. Probable morphotypes of Laccaria spp. were more abundant and frequent in the youngest stand (33\%). Throughout the chronosequence, unique and shared morphotypes were recorded. This work requires further research; however, it shows that stand age is an important factor in the composition of the ECM fungal communities and a key element in forest management plans.
\end{abstract}

Key words: Morphotypes, Pinus, plantation, reforestation, ectomycorrhizal symbiosis, fungal succession.

\section{Resumen}

La actividad forestal en México se sustenta en el aprovechamiento de diversas especies de pinos; una de las más utilizados es Pinus patula. Los hongos ectomicorrízicos (HECM) incrementan la supervivencia de las plántulas, por lo que su uso asegura el éxito de las plantaciones; además, la reforestación con taxa nativos de pino aumenta la diversidad de HECM. Los objetivos de este trabajo fueron comparar la diversidad de HECM asociados a la edad de los rodales y determinar los cambios en la comunidad fúngica a nivel de morfotipos. El estudio se realizó en una plantación con rodales de 2, 5, 7, 9, 14 a 25 y >50 años. Se recolectaron raíces micorrizadas al inicio de la temporada de lluvias, se agruparon de acuerdo con su morfología y se calculó el índice de diversidad, porcentaje de abundancia y frecuencia relativa. Se determinaron 28 morfotipos, tres a nivel de género: Laccaria (2), Tomentella (2) y Cortinarius (1). Se obtuvo una correspondencia positiva entre el número de morfotipos y la edad del arbolado. Los rodales maduros (14-25 y >50 años) fueron más diversos, con respecto a los jóvenes ( 2 a 9 años); a excepción del de 7 años, cuyos valores coincidieron con los rodales maduros. Los morfotipos probables de Laccaria spp. fueron más abundantes y frecuentes en el rodal más joven (33 \%). A través de la cronosecuencia se registraron morfotipos únicos y compartidos. Se concluye que la edad del rodal es un factor importante en la composición de la comunidad HECM, elementos clave en los planes de manejo forestal.

Palabras clave: Morfotipos, Pinus, plantación, reforestación, simbiosis ectomicorrízica, sucesión fúngica.

Fecha de recepción/Reception date: 29 de julio de 2020

Fecha de aceptación/Acceptance date: 3 de marzo de 2021

${ }^{1}$ Universidad Veracruzana, Campus Xalapa. Facultad de Ciencias Agrícolas. México.

2Universidad Veracruzana Centro de Investigación en Micología Aplicada. México.

*Autor por correspondencia; correo-e: dtrejo@uv.mx 


\section{Introducción}

Pinus patula Schiede ex Schltdl. \& Cham. es una de las especies subtropicales más utilizadas para la reforestación en México, con alto valor comercial por su rápido crecimiento y calidad de la madera (Aparicio-Rentería, 2014). La actividad forestal en el país se sustenta en el aprovechamiento de diversas especies de pinos en plantaciones forestales comerciales, lo que representa $5 \%$ de la producción maderable a nivel nacional (López y Caballero, 2018). Debido a este aprovechamiento, dichas plantaciones presentan intervalos cortos destinados a las rotaciones, y esos cambios en las clases de edades (Spake et al., 2015) reducen la riqueza de hongos ectomicorrízicos (HECM) (Kranabetter et al., 2005).

La simbiosis ectomicorrízica tiene un papel importante en la adquisición de agua y nutrientes, principalmente, en especies de la familia Pinaceae; lo que facilita el establecimiento y sobrevivencia de las plántulas (Guo et al., 2020). Las comunidades de HECM están conformadas por ensamblajes de especies que cambian en el espacio y tiempo (Peay et al., 2010). Los cambios en la composición de las comunidades de HECM se relacionan con el desarrollo de la masa forestal; de tal manera que, la edad del rodal es un factor clave en la dinámica de los HECM (Bonet et al., 2004).

Las taxones fúngicos se clasifican en dos patrones de sucesión bajo un modelo de estrategias adaptativas: i) especies de estadios tempranos que prosperan en zonas con alto disturbio y baja intensidad de estrés, como Hebeloma (Fr.) P. Kumm, Lactarius Pers. e Inocybe (Fr.) Fr.; las cuales colonizan las plántulas mediante esporas que permanecen en forma de propágulos resistentes en el suelo (Nara, 2008); y ii) taxones de estadios tardíos, que se encuentran en las raíces más cercanas al tronco, asociados a individuos de mayor edad y tamaño (Nara et al., 2003a); que pertenecen a géneros como Boletus, Russula y Cortinarius, que tienen la habilidad de colonizar nuevos hospederos a través del micelio extrarradical, y algunas especies de Amanita han sido registradas en sitios maduros como cointroducidas (Taylor y Bruns, 1999; VIk et al., 2020). 
Estos patrones de sucesión se han evaluado en función tanto de los esporomas (Jones et al., 2003; Nara et al., 2003a; Fernández-Toirán et al., 2006; Dejene et al., 2017; Gómez-Hernández et al., 2019), como de los morfotipos de raíces micorrizadas (Nara et al., 2003b; Horton et al., 2005; Palfner et al., 2005; Ashkannejhad y Horton, 2006; Peay et al., 2010; Reverchon et al., 2010; Ma et al., 2012; Palacios et al., 2012). Por ejemplo, se observó un incremento en la diversidad de morfotipos en rodales de 30 a 60 años en Picea A. Dietr. (Palfner et al., 2005).

Otros autores señalan un aumento en la diversidad de HECM a nivel de morfotipos en Pseudotsuga menziesii (Mirb.) Franco en rodales de 40 y 400 años (Horton et al., 2005); y en Pinus densiflora Siebold \& Zucc., en arbolado de 55 y 80 años (Ma et al., 2012) a lo largo de una cronosecuencia. Guo et al. (2020) indican que la diversidad de HECM aumentó, significativamente, con la edad del rodal, y la estructura de la comunidad HECM difiere entre los grupos de edad en rodales de Pinus sylvestris var. mongolica Litv. de 26, 33 y 43 años, donde el género Wilcoxina fue el más dominante en los tres grupos de edades.

Especies pertenecientes a las familias Russulaceae y Thelephoraceae son dominantes en estadios sucesionales tardíos de ecosistemas templados, donde juegan un papel crítico en el ciclaje de nutrientes y presentan una mayor adaptación a las condiciones climáticas particulares del sitio (Erland y Taylor, 2002; Koizumi et al., 2018).

Derivado de la reforestación con Pinus spp. en regiones fuera de su área de distribución, algunas especies de HECM se han introducido en áreas en las cuales no está presente su hospedero nativo, o bien los propágulos de HECM nativos existentes son incompatibles con el hospedero introducido. De acuerdo con la revisión de Vellinga et al., (2009) y Vlk et al., (2020), a nivel mundial se han identificado, aproximadamente, 300 especies de HECM introducidas; con al menos 54 géneros de HECM registrados en plantaciones de pinos, entre ellos: Amanita, Astraeus, Boletus, Clavulina, Cortinarius, Gautieria, Gomphidius, Hebeloma, Inocybe, Laccaria, Lactarius, Pisolithus, Ramaria, Russula, Scleroderma, Suillus, Thelephora, y Tricholoma. Taxones pertenecientes a Wicoxina, Suillus, Rhizopogon, Laccaria, 
Pisolithus y Scleroderma tienen adaptaciones ecológicas (ejemplo: mayor tasa de germinación y longevidad de esporas) que les permiten establecerse en estadios tempranos asociados a hospederos de la familia Pinaceace, principalmente.

Las plantaciones con hospederos introducidos presentan baja riqueza de HECM $(<50$ taxa), con respecto a las plantaciones establecidas con hospederos nativos (Ning et al., 2020). En este contexto, Alem et al. (2020) documentan que en una plantación de Pinus patula en Etiopia, con diferentes clases de edades (5, 11 y 36 años), 41 \% de las unidades taxonómicas operativas (OTU, por sus siglas en inglés) de los hongos identificados fueron saprobios, $7 \%$ patógenos y $2 \%$ HECM.

En México, a pesar de que $P$. patula es una especie nativa, poco se ha estudiado sobre los patrones de sucesión fúngica en plantaciones forestales; Gómez-Hernández et al., (2019) identificaron un total de 63 taxa de HECM y 43 saprobios en rodales de 1, 11 y 60 años, con base únicamente en la secuenciación de esporomas; y Ramírez-Miguel et al. (2021) citaron 14 morfotipos HECM en raíces de $P$. patula y $Q$. crassifolia; de ellos, Lactarius sp., Cenococcum geophilum Fr. y Tomentella radiosa (P.Karst.) Rick fueron los más frecuentes en ambos hospederos.

Los análisis basados tanto en la secuenciación de morfotipos y esporomas, como la caracterización morfológica de los mismos permiten entender las estrategias adaptativas de los HECM para establecerse en áreas y hospederos fuera y dentro de su área de distribución. Si bien, la caracterización morfológica de raíces micorrizadas presenta algunas desventajas metodológicas, como el tiempo de evaluación de muestras y la baja identificación a nivel de especie, en contraste con las ventajas que ofrecen las técnicas moleculares sobre este último aspecto; esta técnica se utiliza como una estrategia clave en la producción de planta en vivero, cuando se aplican inoculaciones controladas de HECM (Galindo-Flores et al., 2015), y como un complemento importante en estudios sobre la simbiosis ectomicorrízica.

El objetivo del presente trabajo fue determinar los cambios en la comunidad de HECM a nivel de ectomicorrizas, en rodales de diferentes edades en una plantación establecida de $P$. patula; se destaca la importancia de las plantaciones forestales 
como refugios de gran diversidad fúngica y el alto potencial de estos hongos para ser seleccionados en investigaciones futuras como inoculantes forestales de $P$. patula, lo que favorecerá un mayor éxito de establecimiento en sus plantaciones en sitios dentro y fuera de su área de distribución natural.

\section{Materiales y Métodos}

\section{Sitio de muestreo}

El muestreo se realizó en una plantación de Pinus patula, en la localidad Coxmatla, municipio Xico, Veracruz, (19²5'53.29" N, 97004'47.91" W); a una altitud de 2 209 msnm, con una superficie de 25 ha (Figura 1). La plantación se estableció en 2010 con semilla de la región y planta producida en viveros locales, estos sitios corresponden al rodal de 9 años, cumplidos al 2019 fecha del muestreo. El sitio de 7 años se plantó en 2012, el sitio de 5 años en 2014 y el sitio que representa la edad de 2 años en 2017. Las áreas de 14-25 y de 50 o más años, se establecieron como sitios permanentes de medición y observación, para tomar datos de incrementos y volúmenes de madera. Todos los rodales son puros de $P$. patula y tienen una densidad de 1100 plantas por hectárea a $3 \times 3$ metros. 


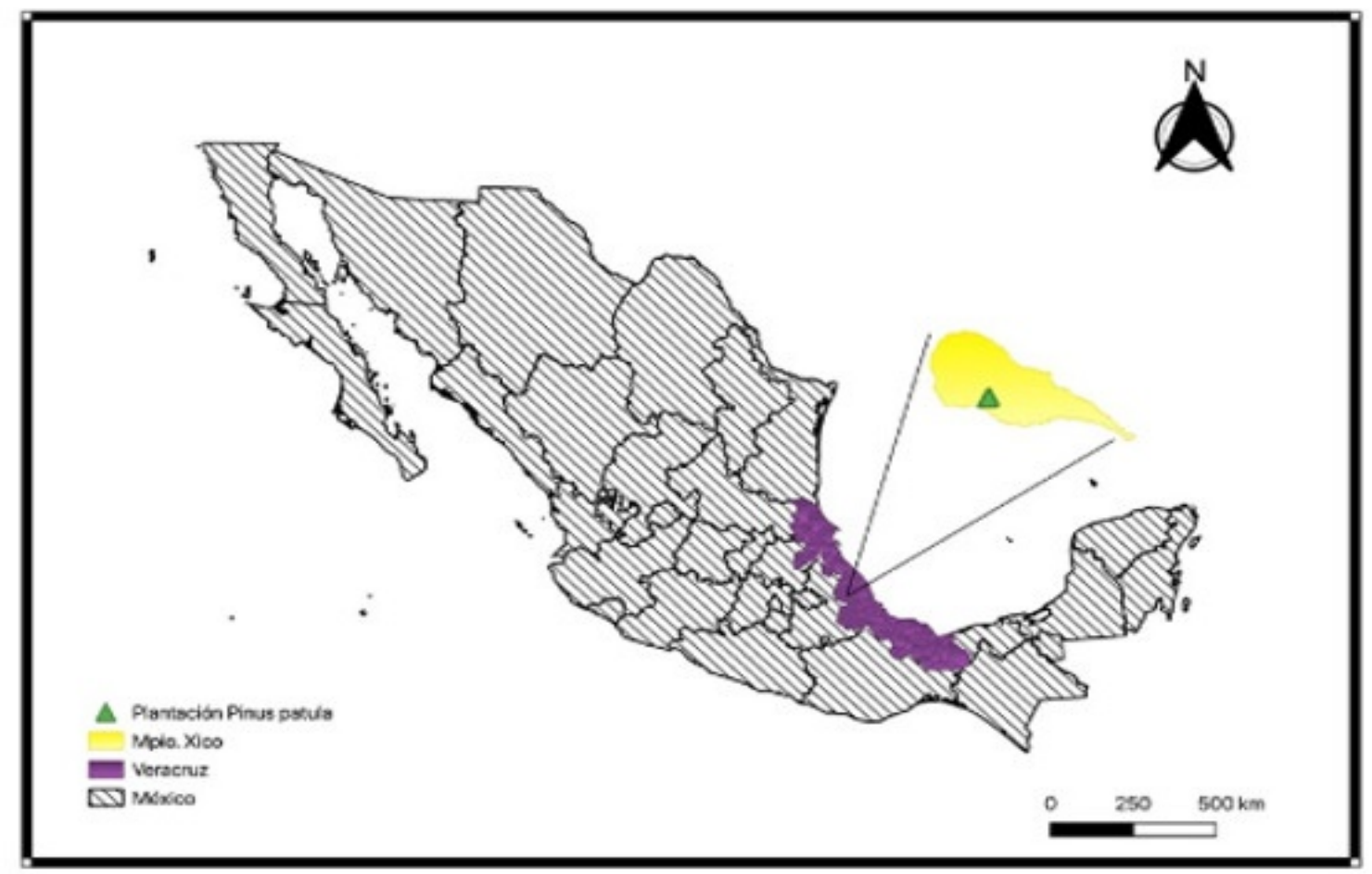

Figura 1. Zona centro del estado de Veracruz, ubicación del sitio de muestreo.

Los sitios de 5, 7 y 9 años para el año 2019 tuvieron un aclareo de $30 \%$. De los rodales de mayor edad solo se han extraído los árboles secos, malformados y algunos suprimidos. El área total de la plantación se caracteriza por poseer relictos naturales de $P$. patula y $P$. pseudostrobus Lindl., con un antecedente de uso de suelo para la ganadería; por lo que, el suelo presenta problemas de compactación y degradación; sin embargo, durante la última década la plantación se ha dedicado para conservación e investigación.

\section{Muestreo de raíces}

Se establecieron seis categorías de edad: $2,5,7,9,14-25$ y >50 años, en cada rodal se recolectaron raíces de 10 árboles, los cuales se seleccionaron al azar (Cuadro 1); el muestreo se realizó durante el mes de julio de 2018. Por cada individuo se obtuvieron tres replicas, en forma de triángulo, a una distancia de $25 \mathrm{~cm}$ del tronco (Guo et al., 
2020, modificado); se removió la materia orgánica y se utilizó un nucleador de metal (AMS) de $5 \mathrm{~cm}$ de diámetro y $20 \mathrm{~cm}$ de profundidad (Figura 2).

Cuadro 1. Descripción de los rodales muestreados.

\begin{tabular}{|c|c|c|c|}
\hline Rodal & $\begin{array}{l}\text { Edad del rodal } \\
\text { (años) }\end{array}$ & $\begin{array}{l}\text { Altitud } \\
\text { (msnm) }\end{array}$ & Coordenadas \\
\hline $\mathrm{R} 1$ & 2 & 2022 & $\begin{array}{l}19^{\circ} 25^{\prime} 53.64120^{\prime \prime} \mathrm{N} \\
97^{\circ} 04^{\prime} 47.77320^{\prime \prime} \mathrm{O}\end{array}$ \\
\hline $\mathrm{R} 2$ & 5 & 2008 & $\begin{array}{l}19^{\circ} 25^{\prime} 52.32720^{\prime \prime} \mathrm{N} \\
97^{\circ} 04^{\prime} 48.11880^{\prime \prime} \mathrm{O}\end{array}$ \\
\hline R3 & 7 & 2005 & $\begin{array}{l}19^{\circ} 25^{\prime} 55.62480^{\prime \prime} \mathrm{N} \\
97^{\circ} 04^{\prime} 42.85920^{\prime \prime} \mathrm{O}\end{array}$ \\
\hline $\mathrm{R} 4$ & 9 & 2018 & $\begin{array}{l}19^{\circ} 25^{\prime} 50.71080^{\prime \prime} \mathrm{N} \\
97^{\circ} 04^{\prime} 48.01800^{\prime \prime} \mathrm{O}\end{array}$ \\
\hline R5 & $14-25$ & 2028 & $\begin{array}{l}19^{\circ} 25^{\prime} 58.03680^{\prime \prime} \mathrm{N} \\
97^{\circ} 04^{\prime} 37.15680^{\prime \prime} \mathrm{O}\end{array}$ \\
\hline R6 & $>50$ & 2014 & $\begin{array}{l}19^{\circ} 25^{\prime} 53.90400^{\prime \prime} \mathrm{N} \\
97^{\circ} 04^{\prime} 41.40120^{\prime \prime} \mathrm{O}\end{array}$ \\
\hline
\end{tabular}

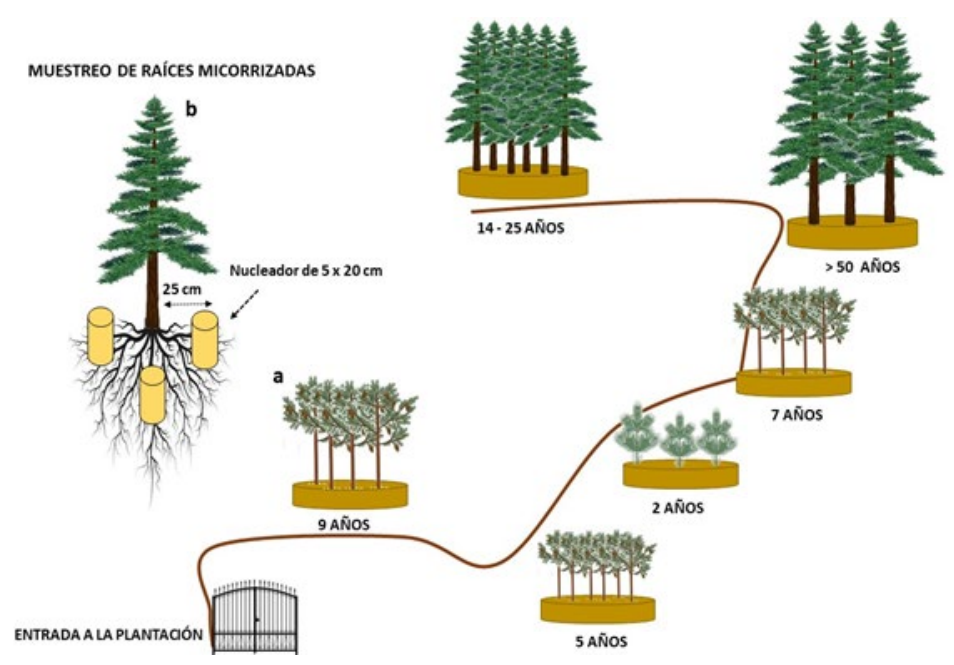

Figura 2. Distribución de los rodales dentro de la plantación de Pinus patula Schiede ex Schltdl. \& Cham. (a); toma de muestras (b). 
Se extrajeron las puntas micorrizadas de cada muestra. Las raíces más grandes, se separaron, las restantes se lavaron y tamizaron con un tamiz de $1 \mathrm{~mm}$ y otro de $2 \mathrm{~mm}$, estas se disectaron bajo un microscopio estereoscópico Leica EZ4, y separaron aquéllas con micorrizas turgentes y con manto, de las muertas o no micorrizadas. Se limpiaron cuidadosamente con agua destilada. Se describieron, macroscópicamente, los caracteres morfológicos de las micorrizas para separarlas en morfotipos según sus caracteres morfológicos, con base en la metodología estándar de Agerer (1991): tipo de ramificación, color, ramificación de las puntas, color del manto, rizomorfos e hifas emanentes. Cada morfotipo se fotografió con una cámara Motorola G8 con un aumento de 40X.

\section{Análisis estadísticos}

Se estimó la riqueza e índices de diversidad de Shannon-Wiener ( $\left.H^{\prime}\right)$ y Simpson (D), estas variables se compararon entre las diferentes edades de los rodales mediante un modelo lineal generalizado, comparaciones LSD Fisher, con nivel de significancia de $95 \%$; previa comprobación de los supuestos de normalidad y heterogeneidad de varianza en los datos; se utilizaron los programas Statistica 9.0 (StatSoft, Inc.), EstimateS 9.1.0, PAST (Hammer et al., 2001).

La abundancia relativa se calculó dividiendo el número de raíces micorrizadas por morfotipo entre el total de raíces micorrizadas para cada rodal. La frecuencia relativa se estimó como la frecuencia de cada morfotipo dividido entre la suma de las frecuencias de todos los morfotipos presentes por rodal. Se determinó la influencia de la edad del rodal en la distribución de los morfotipos de acuerdo con su presencia y abundancia, mediante un análisis de correspondencia, sin tendencia (DCA). Se construyó un Diagrama de Venn para comparar los morfotipos compartidos y exclusivos por rodal. 


\section{Resultados}

Se evaluaron 1849 puntas micorrizadas, obtenidas de un total de 30 muestras por rodal estudiado, las cuales correspondieron morfológicamente a 28 morfotipos. Se identificaron, tentativamente, cinco morfotipos a nivel de género: Cortinarius sp., Laccaria spp., y Tomentella spp. (Figura 3), los restantes no se lograron identificar debido a la falta de características diagnósticas.
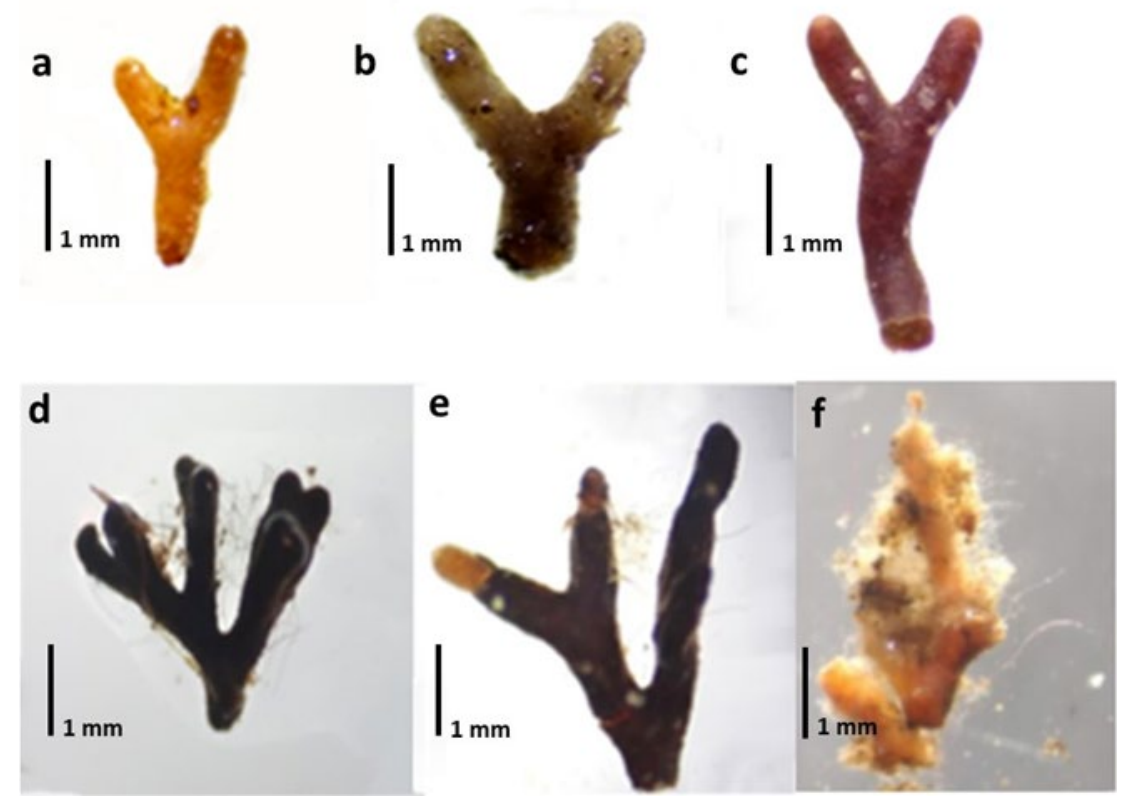

(a) Laccaria spp.; (b, c) Morfotipo no identificado (M6, M7); (d, e) Tomentella spp., (f) Cortinarius sp. (barra de escala $=1 \mathrm{~mm}$ ).

Figura 3. Morfología de las ectomicorrizas más frecuentes y con probable identificación en una cronosecuencia de Pinus patula Schiede ex Schltdl. \& Cham.

El rodal con mayor riqueza de morfotipos fue R5 con 14, los que correspondieron a $50 \%$ del total registrado; seguido de R6, con 9 morfotipos (32.1\%); R3 con 8 morfotipos (28.5\%); R2 con 7 morfotipos (25\%); R4 con 6 morfotipos (21.4 \%); y R1 con 5 morfotipos (17.8 \%) (Figura 4, Cuadro 2). 

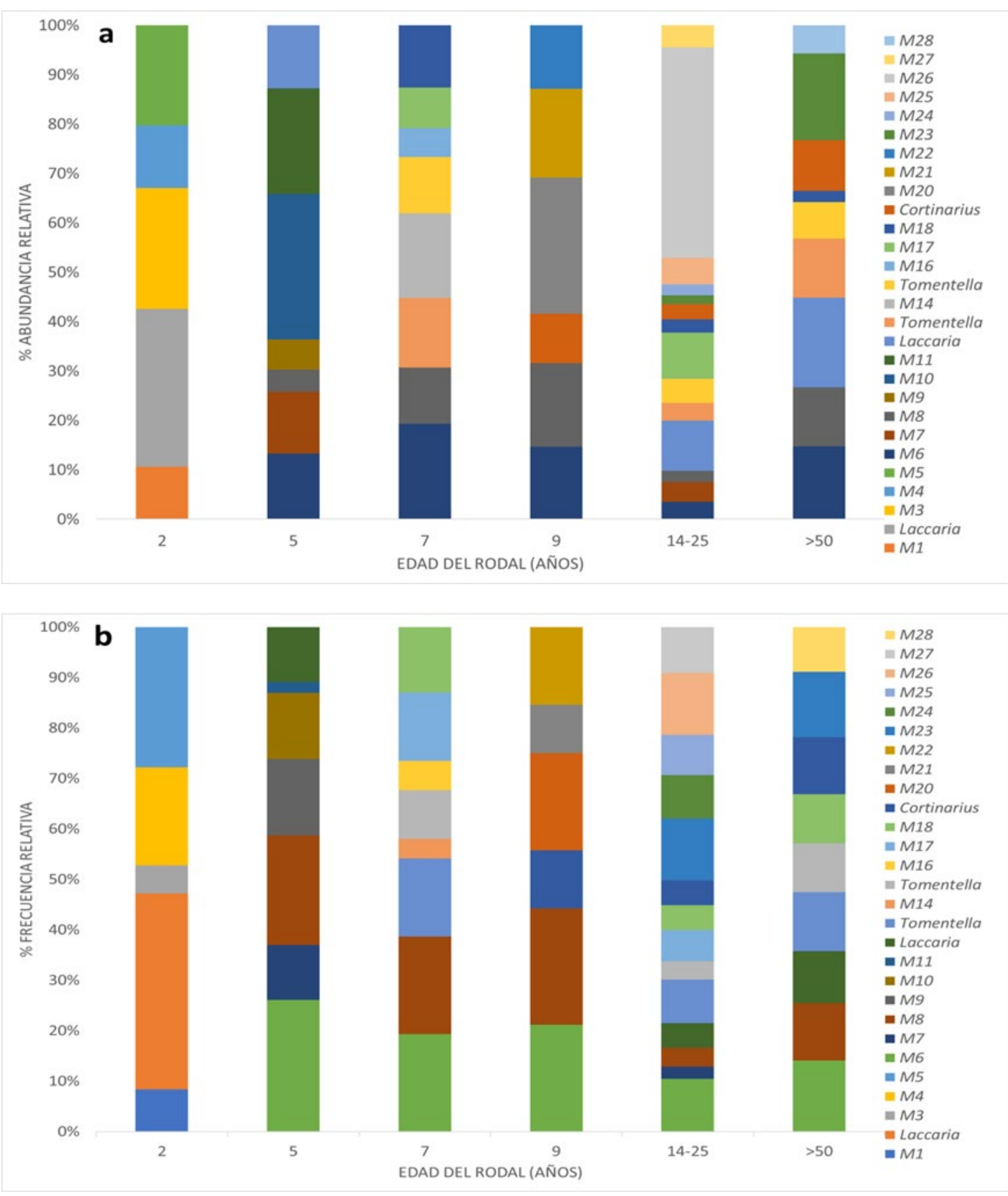

Figura 4. Abundancia relativa (a) y Frecuencia relativa (b) de morfotipos de HECM en seis rodales de diferentes edades de Pinus patula Schiede ex Schltdl. \& Cham. 
Cuadro 2. Índices de a-diversidad de la comunidad de HECM (basado en morfotipos) en los rodales de Pinus patula Schiede ex Schltdl. \& Cham. de diferentes edades.

\begin{tabular}{|c|c|c|c|c|c|}
\hline Rodal & $\begin{array}{l}\text { Número de } \\
\text { morfotipos }\end{array}$ & $\begin{array}{l}\text { Total de puntas } \\
\text { micorrizadas } \\
\text { analizadas }\end{array}$ & Morfotipos & $\begin{array}{l}\text { Simpson } \\
\left(D^{\prime}\right)\end{array}$ & $\begin{array}{c}\text { Shannon } \\
\text { Wiener } \\
\left(\mathrm{H}^{\prime}\right)\end{array}$ \\
\hline $\mathrm{R} 1$ & 5 & $94_{c}$ & M1, Laccaria spp., M3, M4, M5 & $0.76 \pm 0.04$ & $1.53 \pm 0.07 \mathrm{~d}$ \\
\hline $\mathrm{R} 2$ & 7 & $360_{b}$ & $\begin{array}{l}\text { M6, M7, M8, M9, M10, M11, } \\
\text { Laccaria spp. }\end{array}$ & $0.81 \pm 0.06$ & $1.79 \pm 0.02_{c}$ \\
\hline R3 & 8 & $326_{b c}$ & $\begin{array}{c}\text { M6, M8, Tomentella spp., M14, } \\
\text { M16, M17, M18 }\end{array}$ & $0.86 \pm 0.08$ & $2.02 \pm 0.05 b$ \\
\hline R4 & 6 & $668 \mathrm{a}$ & $\begin{array}{c}\text { M6, M8, Cortinarius sp., M20, } \\
\text { M21, M22 }\end{array}$ & $0.81 \pm 0.05$ & $1.74 \pm 0.02_{c}$ \\
\hline R5 & 14 & $225 b c$ & $\begin{array}{c}\text { M6, M7, M8, Laccaria spp., } \\
\text { Tomentella spp., M17, M18, } \\
\text { Cortinarius sp., M23, M24, } \\
\text { M25, M26, M27 }\end{array}$ & $0.78 \pm 0.04$ & $2.07 \pm 0.06 \mathrm{a}$ \\
\hline R6 & 9 & $176_{\mathrm{bc}}$ & $\begin{array}{l}\text { M6, M8, Laccaria spp., } \\
\text { Tomentella spp., M18, } \\
\text { Cortinarius sp., M23, M28 }\end{array}$ & $0.86 \pm 0.06$ & $2.08 \pm 0.05 a$ \\
\hline
\end{tabular}

Los valores de a-diversidad representan los promedios por cada 30 muestras por rodal (tres repeticiones por cada árbol por rodal). Las letras representan las diferencias significativas $(P<0.05)$.

Se observaron diferencias significativas en la abundancia de HECM respecto a la edad de los rodales $(P=0.002)$. La mayor abundancia total de ápices micorrizados se presentó en R4, con 36 \% (668); seguido de R2, con $19.4 \%$ (360); R3 con $17.6 \%$ (326); R5 con $12.16 \%$ (225); R6 con $9.5 \%$ (176); y R1 con $5.08 \%$ (94) (Figura 4).

El morfotipo probable de Laccaria spp. registró una abundancia relativa ( $A i)$ de $33.9 \%$ en el rodal de 2 años (R1) y una frecuencia relativa (Fi) de $38.8 \%$. En R2, el morfotipo M10 mostró el mayor valor de $A i(29.44 \%)$ y $13.04 \%$ de Fi. Los morfotipos M6 ( $A i=19.3 \%)$ y M14 ( $A i=17.1 \%)$ tuvieron los valores más altos en R3; aunque, M14 presentó una frecuencia relativa de $3.8 \%$, el más bajo para este rodal. En R4, M20 tuvo un valor alto de $A i(27.5 \%)$, con una $\mathrm{Fi}$ de $19.23 \%$. Los rodales R5 y R6 compartieron una gran cantidad de morfotipos, con una dominancia del morfotipo 
M26 en el R5, cuya $A i$ fue de $41.9 \%$ y una Fi de $12.28 \%$. En R6, la abundancia y frecuencia relativa para cada morfotipo fue similiar. Los morfotipos M6 y M8 se identificaron en todos los rodales, excepto en R1 ( 2 años), en los que contribuyeron con $23.4 \%$ de la abundancia total (Figura 4).

Los índices de a-diversidad (Cuadro 2) indicaron un incremento de la diversidad en relación con la edad del rodal; los rodales de mayor edad R5 y R6 $\left(H^{\prime}=2.07 \pm 0.06\right.$, $\left.H^{\prime}=2.08 \pm 0.05\right)$ presentaron valores superiores en comparación con los más jóvenes, a excepción del R3, cuyo índice $H^{\prime}$ fue similar al estimado para los rodales R5 y R6.

El análisis de correspondencia sin tendencia (DCA) muestra la distribución de todos los morfotipos de HECM en las diferentes edades de los rodales, y explicó $78 \%$ de la variabilidad de los datos en los ejes $2(64 \%)$ y 3 (14\%) (Figura 5). En el gráfico se presenta la distribución de los morfotipos y los divide en cuatro grupos: G I consta de los morfotipos compartidos en R3, R5 y R6 entre los cuales se observan muchas unidades entremezcladas, lo que hace suponer una alta similitud entre sus ensamblajes de morfotipos de HECM. El segundo grupo (G II), lo compone M1, Laccaria spp., M3, M4 y M5 en el rodal de 2 años, los cuales no se encontraron en los otros rodales. Un tercer grupo (G III), con M7, M9, M10 y M11 presentes únicamente en el rodal de 5 años; y un cuarto grupo (G IV) de morfotipos distribuidos en el rodal de 14-25 años (R5). 


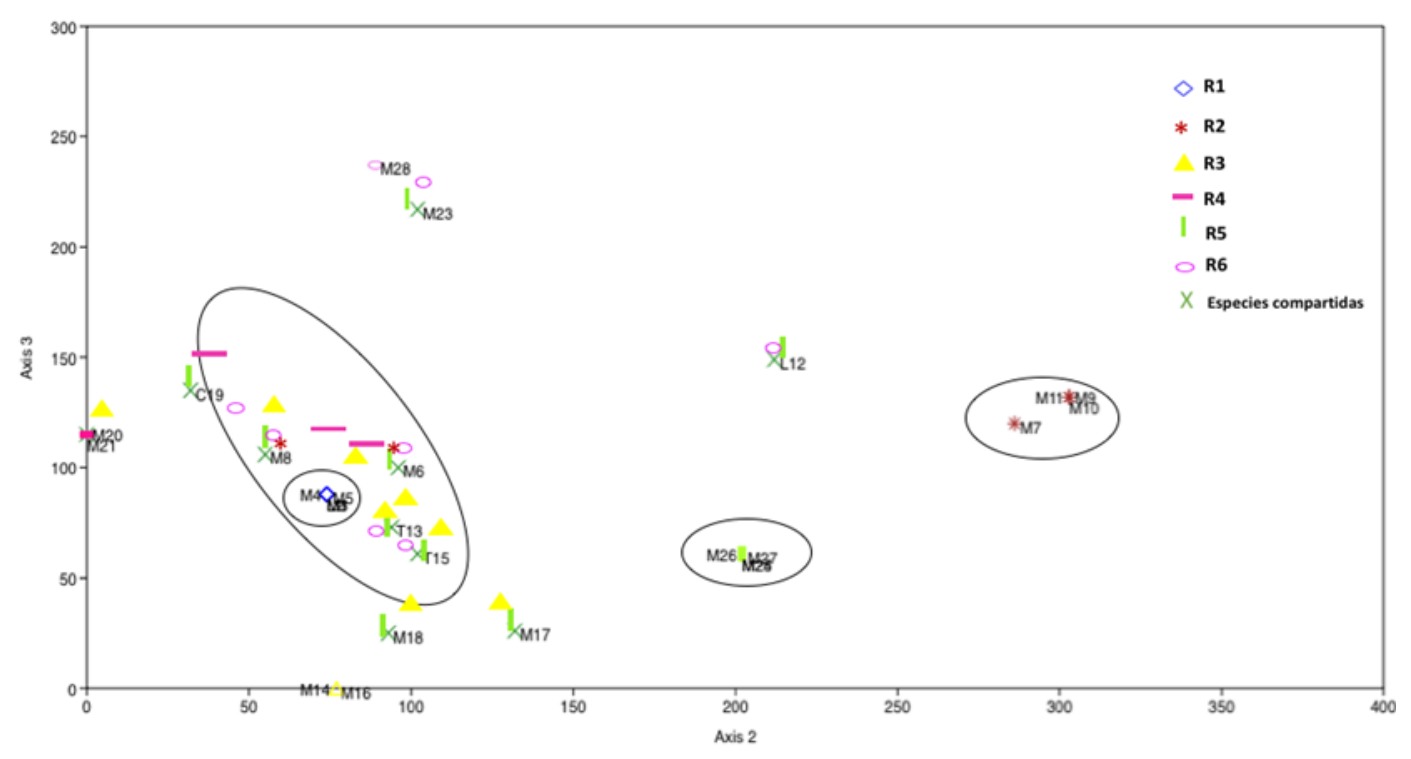

Figura 5. Análisis de correspondencia sin tendencia (DCA) para los morfotipos de HECM presentes en seis rodales de diferentes edades en una plantación de Pinus patula Schiede ex Schltdl. \& Cham.

A lo largo de la cronosecuencia se registraron morfotipos únicos y compartidos. Los morfotipos M6 y M8 se observaron en todos los rodales, a excepción del R1, el cual no tuvo morfotipos compartidos con el resto de los rodales. En R5 se obtuvo la cantidad más grande de morfotipos únicos (4); mientras que en R6 solo hubo un morfotipo único (M28). Los rodales de mayor edad ( $R 5$ y R6) compartieron un mayor número de morfotipos (Figura 6). 


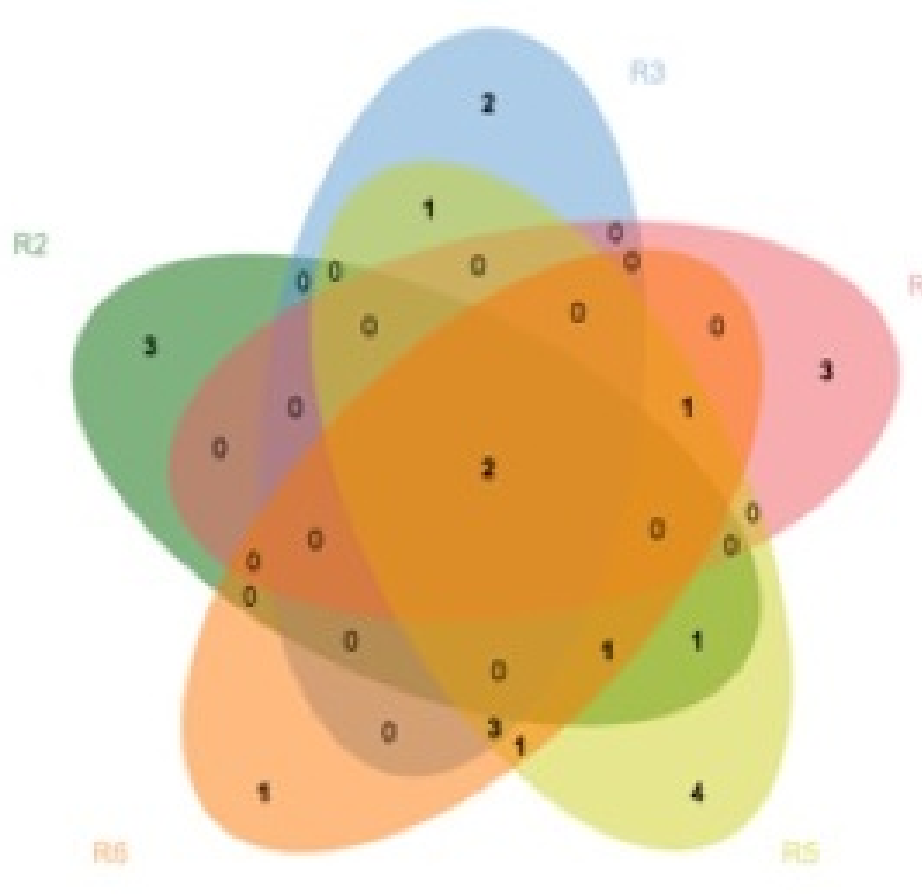

Figura 6. Morfotipos compartidos entre los rodales de diferentes edades de Pinus patula Schiede ex Schltdl. \& Cham. Se excluye el rodal de 2 años (R1) ya que no comparte ningún morfotipo con el resto de los rodales.

\section{Discusión}

Los índices de a-diversidad mostraron diferencias entre las edades de los rodales. Se esperaba que la diversidad a nivel de morfotipos aumentara de acuerdo con la edad del rodal; y aunque, hubo una tendencia de incremento en los rodales de 14-25 y >50 años, los resultados evidenciaron valores de diversidad similares en el rodal de 7 años. Esto, puede explicarse debido a que el rodal de 7 años ha tenido un aclareo de $30 \%$, lo que permitió mayor entrada de luz a las plantas del sotobosque, las cuales contribuyen a mejorar la disponibilidad de agua y nutrientes en suelo (Dang et al., 2018).

El rodal de 2 años aún no recibe el primer aclareo y presenta una mayor densidad de árboles por lo tanto menor entrada de luz, lo que redundaría en el bajo número de morfotipos. Gómez-Hernández et al. (2019) observaron un patrón similar en estadios 
intermedios de desarrollo en plantaciones de 11 años de P. patula y Quercus rugosa Neé, donde la diversidad de especies HECM fue similar a los rodales maduros (60 años) con una composición arbórea mixta ( $P$. patula, P. douglasiana Martínez, $P$. teocote Schiede ex Schltdl. \& Cham., Q. rugosa y Q. laurina Bonpl.); mientras que, disminuyen en rodales jóvenes ( 1 año) exclusivos de $P$. patula; los autores señalan que el desarrollo del dosel y la densidad de árboles son factores importantes en la producción de esporomas y en el aumento en la diversidad de HECM.

Los resultados del presente estudio, si bien son exploratorios, muestran que los procesos de sucesión influyen directamente en los HECM y esa diversidad está modulada por el tiempo de establecimiento de la plantación. Los rodales de mayor edad tienden a ofrecer mejores hábitats para las especies de HECM que los más jóvenes y heterogéneos; en contraste con lo citado por Dejene et al. (2017) en sitios donde el género Pinus es una especie introducida, se registran pocas especies HECM y baja diversidad asociada a P. patula; lo anterior debido a la poca cantidad de propágulos compatibles en suelo. Estos autores señalan que no encontraron especies de HECM en rodales de 5 años, pero citan un incremento de esporomas HECM en rodales de 11 a 36 años.

Alem et al. (2020) sugieren que el aclareo y el uso previo de suelo son los principales factores que modulan las comunidades edáficas de HECM en $P$. patula. Estos autores sugieren que rodales de 5 y 36 años son más diversos en cuanto a especies HECM, en comparación con rodales intermedios de 11 años, lo que se debe al poco desarrollo del dosel y a la posibilidad de que el sitio fuese utilizado, previamente, para fines agrícolas; por ello, presenta alto número de propágulos asociados a estadios jóvenes procedentes del banco de esporas de las plantaciones adyacentes. Lo mismo se ha observado en otras coníferas plantadas en zonas fuera de su intervalo de distribución natural, como Pinus radiata D. Don, especie estudiada en rodales de 20 años, en los cuales se registra un total de 11 morfotipos y una baja diversidad de HECM, en comparación con los rodales de 5 y 10 años, estos últimos con menor densidad del dosel (Palacios et al., 2012). 
Los valores más altos de diversidad de especies HECM se encontraron en los rodales de 14-25 años y >50 años, lo que refleja la diferencia entre las intervenciones silvícolas aplicadas, Parladé et al. (2017) y Tomao et al. (2017) mencionan que la intensidad parcial de aclareo es un factor que favorece la aparición de esporomas HECM y pero no necesariamente afecta la diversidad de especies a nivel de raíces (Castaño et al., 2018), sin embargo, este es parámetro no ha sido evaluado en el estudio de manera directa pero es necesario considerarlo bajo diferentes condiciones de suelo y climáticas para modelar tendencias en cuanto a su efecto sobre otros grupos funcionales de la diversidad fúngica.

En la Figura 5, se observa que, en los rodales de 7, 14- 25 y >50 años la mayoría de los morfotipos se comparten, lo que supone que no necesariamente la edad del rodal determina, tanto la diversidad como la composición de la comunidad HECM. Castaño et al. (2019) señalan que la diversidad y abundancia de las especies HECM se incrementan con la edad del árbol; pero son afectadas de manera directa por la disponibilidad de $\mathrm{N}$ y $\mathrm{P}$, así como por el aumento de la relación $\mathrm{C} / \mathrm{N}$.

En el presente trabajo se registró un alto número de morfotipos de HECM asociados a una plantación de $P$. patula, mediante el uso de la caracterización morfológica de las ectomicorrizas, dicho método tiene limitaciones, por la dificultad para la diferenciación entre especies, lo que genera la subestimación o sobrestimación de la riqueza de especies asociadas a un hospedero. Indiscutiblemente, los métodos moleculares son útiles en la identificación de estas comunidades HECM, ya permiten clarificar la identidad de los taxones de hongos; sin embargo los métodos morfológicos representan una primera aproximación en la diferenciación de patrones de recambio y dominancia de las especies asociadas a los hospederos, de los cuales, cabe señalar, se tiene poca o nula información; tal es el caso de $P$. patula, taxón con un alto potencial de aprovechamiento forestal dentro y fuera de su intervalo de distribución natural.

En México se han descrito algunos morfotipos en estadios jóvenes de $P$. patula: Boletus edullis Bull., Laccaria bicolor (Maire) P.D. Orton, L. proxima (Boud.) Pat., Hebeloma alpinum 
(J. Favre) Bruchet, H. leucosarx P.D. Orton, H. mesophaeum (Pers.) Quél y Suillus pseudobrevipes A.H. Sm. \& Thiers (Carrera-Nieva y López-Ríos, 2004; Carrasco-Hernández et al., 2010; Jiménez, 2011), principalmente, en experimentos con inoculación controlada; sin embargo, este es una de las primeras investigaciones en las que se realiza una exploración a nivel morfotipos de las raíces micorrizadas, dentro de una cronosecuencia en rodales del mismo hospedero; aunque es importante implementar futuros trabajos a nivel molecular (Ramírez-Miguel et al., 2021).

Los hongos ectomicorrízicos cumplen un papel estructural que determina las condiciones para el establecimiento y desarrollo de distintas especies forestales (PérezMoreno et al., 2020), por lo que es fundamental la evaluación de la diversidad fúngica en bosques de plantación desde la perspectiva de la compresión de prácticas silviculturales, como talas parciales, aclareos de baja intensidad (30-50 \%), la mezcla de variedades vegetales nativas y el desarrollo de los rodales que promuevan la interconectividad dentro de la matriz forestal y aseguren el establecimiento de especies HECM pioneras.

\section{Conclusiones}

La diversidad de morfotipos de HECM está relacionada con la edad del rodal, aunque no es el único factor. Los valores más altos de diversidad se encontraron en rodales de 7, 14-25 y >50 años. La composición de la comunidad de HECM está agrupada en morfotipos únicos en el rodal más joven y ectomicorrizas similares entre los sitios de $7,9,14-25$ y $>50$ años. Se determinaron los morfotipos HECM más abundantes y frecuentes en raíces de $P$. patula, describiendo a Laccaria como uno de los más abundantes en los sitios más jóvenes. Este trabajo resalta la importancia de las plantaciones forestales como refugios de diversidad fúngica. 


\section{Agradecimientos}

Los autores agradecen al Laboratorio de Organismos Benéficos de la Universidad Veracruzana por facilitar los medios para el procesamiento de las muestras. A J. Dorantes por su valioso apoyo en el trabajo de campo y las facilidades brindadas. Todos los autores agradecen a los revisores y al editor por sus oportunos comentarios que contribuyeron a la mejora del manuscrito.

\section{Conflicto de intereses}

Los autores declaran no tener conflicto de intereses.

\section{Contribuciones de los autores}

Yajaira Baeza Guzmán y Dora Trejo Aguilar: diseño de estudio, realización de la disección de raíces, análisis estadísticos e interpretación de los resultados; Jesús Dorantes López: establecimiento de las plantaciones de Pinus patula, lo que permitió hacer este estudio, al crear un laboratorio natural. Yajaira Baeza Guzmán, Rosario Medel Ortiz y Dora Trejo Aguilar: redacción y revisión del manuscrito final.

\section{Referencias}

Agerer, R. 1991. Characterization of ectomycorrhiza. Methods in microbiology 23: 25-73. Doi: 10.1016/S0580-9517(08)70172-7.

Alem, D., T. Dejene, J. A. Oria-de-Rueda, J. Geml and P. Martín-Pinto. 2020. Soil Fungal Communities under Pinus patula Schiede ex Schltdl. \& Cham. Plantation Forests of Different Ages in Ethiopia. Forests 11(10): 1109. Doi: 10.3390/f11101109. 
Aparicio-Rentería, A., S. F. Juárez-Cerrillo y L. R. Sánchez-Velásquez. 2014. Propagación por enraizamiento de estacas y conservación de árboles plus extintos de Pinus patula procedentes del norte de Veracruz, México. Madera y Bosques 20 (1): 85-96. Doi: 10.21829/myb.2014.201178.

Ashkannejhad, S. and T. R. Horton. 2006. Ectomycorrhizal ecology under primary succession on coastal sand dunes: interactions involving Pinus contorta, suilloid fungi and deer. New Phytologist 169 (2): 345-354. Doi: 10.1111/j.1469-8137.2005.01593.x.

Bonet, J. A., C. R. Fischer and C. Colinas. 2004. The relationship between forest age and aspect on the production of sporocarps of ectomycorrhizal fungi in Pinus sylvestris forests of the central Pyreness. Forest Ecology and Management 203: 157-175. Doi: 10.1016/j.foreco.2004.07.063.

Carrasco-Hernández, V., J. Pérez-Moreno, V. Espinosa-Hernández, J. J. AlmarazSuárez, R. Quintero-Lizaol y M. Torres-Aquino. 2010. Caracterización de micorrizas establecidas entre dos hongos comestibles silvestres y pinos nativos de México. Revista Mexicana de Ciencias Agrícolas 1(4): 567577.http://www.scielo.org.mx/scielo.php?script=sci_arttext\&pid=S200709342010000400009\&lng=es\&tIng=es. (20 de junio de 2020).

Carrera-Nieva, A. y G. F. López-Ríos. 2004. Manejo y evaluación de ectomicorrizas en especies forestales. Revista Chapingo. Serie Ciencias Forestales y del Ambiente 10 (2): 9398. https://www.redalyc.org/articulo.oa?id=629/62910204 (20 de junio de 2020).

Castaño, C., J. G. Alday, B. D. Lindahl, J. Martínez de Aragón, S. de-Miguel, C. Colinas, J. Parladé, J. Pera and J. A. Bonet. 2018. Lack of thinning effects over inter-annual changes in soil fungal community and diversity in a Mediterranean pine forest. Forest Ecology and Management 424:420-427.

Doi: $10.1016 /$ j.foreco.2018.05.004. 
Castaño, C., T. Dejene, O. Mediavilla, J. Geml, J. A. Oria-de-Rueda and P. MartínPinto. 2019. Changes in fungal diversity and composition along a chronosequence of Eucalyptus grandis plantations in Ethiopia. Fungal ecology 39: 328-335.

Doi: 10.1016/j.funeco.2019.02.003.

Dang, P., Y. Gao, J. Liu, S. Yu and Z. Zhao. 2018. Effects of thinning intensity on understory vegetation and soil microbial communities of a mature Chinese pine plantation in the Loess Plateau. Science of the Total Environment 630: 171-180. Doi: 10.1016/j.scitotenv.2018.02.197.

Dejene, T., J. A. Oria-de-Rueda and P. Martín-Pinto. 2017. Fungal diversity and succession following stand development in Pinus patula Schiede ex Schltdl. \& Cham. plantations in Ethiopia. Forest Ecology and Management 395: 9-18. Doi: 10.1016/j.foreco.2017.03.032.

Erland, S. and A. F. Taylor. 2002. Diversity of ectomycorrhizal fungal communities in relation to the abiotic environment. In: van der Heijden, M. G. A. and I. R. Sanders (eds.). Mycorrhizal ecology. Springer. Heidelberg, Berlin. pp. 163-200. Doi: $10.1007 / 978-3-540-38364-2$.

Fernández-Toirán, L. M., T. Ágreda and J. M. Olano. 2006. Stand age and sampling year effect on the fungal fruit body community in Pinus pinaster forests in central Spain. Botany 84 (8): 1249-1258. Doi: 10.1139/b06-087.

Galindo-Flores, G., C. Castillo-Guevera, A. Campos-López y C. Lara. 2015. Caracterización de las ectomicorrizas formadas por Laccaria trichodermophora y Suillus tomentosus en Pinus montezumae. Botanical Sciences 93 (4): 855-86. Doi: 10.17129/botsci.200.

Gómez-Hernández, M., K. G. Ramírez-Antonio and E. Gándara. 2019. Ectomycorrhizal and wood-decay macromycete communities along development stages of managed Pinus patula stands in Southwest Mexico. Fungal ecology 39:109-116. Doi: 10.1016/j.funeco.2018.12.007. 
Guo, M. S., G. D. Ding, G. L. Gao, Y. Zhang, H. Y. Cao and Y. Re. 2020. Community composition of ectomycorrhizal fungi associated with Pinus sylvestris var. mongolica plantations of various ages in the Horqin Sandy Land. Ecological Indicators 110: 105860. Doi: 10.1002/ece3.6119.

Hammer, Ø., D. A. Harper and P. D. Ryan. 2001. PAST: Paleontological statistics software package for education and data analysis. Palaeontologia electronica 4 (1): 9 http://palaeoelectronica.org/2001_1/past/issue1_01.htm (11 de febrero de 2020).

Horton, T. R., R. Molina y K. Hood. 2005. Douglas-fir ectomycorrhizae in 40-and 400-year-old stands: mycobiont availability to late successional western hemlock. Mycorrhiza 15 (6):393-403. Doi: 10.1007/s00572-004-0339-9.

Jiménez R., M. 2011. Estudio etnomicológico y biotecnológico de hongos silvestres comestibles ectomicorrízicos en Pinus pseudostrobus y evaluación de bacterias promotoras de crecimiento vegetal. Tesis de Maestría. Colegio de Posgraduados. Montecillo, Edo. de Méx., México. 120 p.

Jones, M. D., D. M. Durall and J. W. Cairney. 2003. Ectomycorrhizal fungal communities in young forest stands regenerating after clearcut logging. New Phytologist 157 (3): 399-422. Doi: 10.1046/j.1469-8137.2003.00698.x

Koizumi, T., M. Hattori and K. Nara. 2018. Ectomycorrhizal fungal communities in alpine relict forests of Pinus pumila on Mt. Norikura, Japan. Mycorrhiza 28(2): 129145. Doi: $10.1007 / \mathrm{s} 00572-017-0817-5$.

Kranabetter, J. M., J. Friesen, S. Gamiet and P. Kroeger. 2005. Ectomycorrhizal mushroom distribution by stand age in western hemlock-lodgepole pine forests of northwestern British Columbia. Canadian Journal of Forest Research 35 (7): 15271539. DOI: $10.1139 / \times 05-095$. 
López L., M. Á. y M. Caballero D. 2018. Análisis financiero de una plantación de Pinus patula Schiede ex Schltdl. et Cham. de pequeña escala. Revista Mexicana de Ciencias Forestales 9(46):186-208. DOI: 10.29298/rmcf.v9i46.116.

Ma, D., Z. Shuying, W. Luhe and Z. Dongyou. 2012. Ectomycorrhiza community structure in chronosequences of Pinus densiflora in eastern China. African Journal of Microbiology Research 6 (32): 6204-6209. Doi: 10.5897/AJMR12.902.

Nara, K., H. Nakaya and T. Hogetsu. 2003a. Ectomycorrhizal sporocarp succession and production during early primary succession on Mount Fuji. New Phytologist 158: 193e206. Doi: 10.1046/j.1469-8137.2003.00724.x.

Nara, K., H. Nakaya, B. Y. Wu, Z. H. Zhou and T. Hogetsu. 2003b. Underground primary succession of ectomycorrhizal fungi in a volcanic desert on Mount Fuji. New Phytologist 159: 743e756. Doi: 10.1046/j.1469-8137.2003.00844.x.

Nara, K. 2008. Community developmental patterns and ecological functions of ectomycorrhizal fungi: implications from primary succession. In: Varma, A. (ed.). Mycorrhiza. Springer. Heidelberg, Berlin. pp. 581-599. Doi: 10.1007/978-3-540-78826-3_28.

Ning, C., W. Xiang, G. M. Mueller, L. Egerton-Warburton, W. Yan and S. Liu. 2020. Differences in ectomycorrhizal community assembly between native and exotic pines are reflected in their enzymatic functional capacities. Plant and Soil, 446(1): 179-193. Doi: 10.1007/s11104-019-04355-9.

Palacios, Y., G. Palfner y C. Hernández. 2012. Comunidad ectomicorrícica en una cronosecuencia de Pinus radiata (Pinophyta: Pinaceae) de la zona de transición climática mediterráneo-templada de Chile central. Revista Chilena de Historia Natural 85 (1): 61-71. Doi: 10.4067/S0716-078X2012000100005.

Palfner, G., M. A. Casanova-Katny and D. J. Read. 2005. The mycorrhizal community in a forest chronosequence of Sitka spruce Picea sitchensis (Bong.) Carr. in Northern England. Mycorrhiza 15 (8): 571-579. Doi: 10.1007/s00572-005-0364-3. 
Parladé, J., F. Martínez-Peña and J. Pera. 2017. Effects of forest management and climatic variables on the mycelium dynamics and sporocarp production of the ectomycorrhizal fungus Boletus edulis. Forest Ecology and Management 390: 73-79. Doi: $10.1016 /$ j.foreco.2017.01.025.

Peay, K. G., P. G. Kennedy and T. D. Bruns. 2010. Rethinking ectomycorrhizal succession: are root density and hyphal exploration types drivers of spatial and temporal zonation. Fungal ecology 4 (3): 233-240. Doi: 10.1016/j.funeco.2010.09.010.

Pérez-Moreno, J., M. Martínez-Reyes and F. Hernández-Santiago.2020. Biotechnology of inoculation of trees of forest importance with edible ectomycorrhizal fungi Agroproductividad 13(5): 91-93. Doi 10.32854/agrop.vi.1540.

Ramírez-Miguel, A. A., A. F. Hernández-Díaz, C., Valenzuela-Encinas, R. Garibay-Orijel y C. Truong. 2021. Hongos ectomicorrízicos asociados a plantas jóvenes de Pinus patula y Quercus crassifolia en plantaciones del sistema matarrasa de la Sierra Juárez de Oaxaca, México. Scientia Fungorum 1-13. Doi: 10.33885/sf.2021.51.1289.

Reverchon, F., P. Ortega-Larrocea, J. Pérez-Moreno, V. Peña-Ramírez and C. Siebe. 2010. Changes in community structure of ectomycorrhizal fungi associated with Pinus montezumae across a volcanic soil chronosequence at Sierra Chichinautzin, Mexico. Canadian journal of forest Research 40 (6): 1165-1174. Doi: 10.1139/X10-062.

Spake, R., T. H. Ezard, P. A. Martin, A. C. Newton and C.P. Doncaster. 2015. A meta-analysis of functional group responses to forest recovery outside of the tropics. Conservation Biology 29 (6): 1695-1703. Doi: 10.1111/cobi.12548.

Taylor, D. L. and T. D. Bruns. 1999. Community structure of ectomycorrhizal fungi in a Pinus muricata forest: minimal overlap between the mature forest and resistant propagule communities. Molecular Ecology 8(11): 1837-1850. Doi: 10.1046/j.1365-294x.1999.00773.x. 
Tomao, A., J. A. Bonet, J. M. de Aragón and S. de-Miguel. 2017. Is silviculture able to enhance wild forest mushroom resources? Current knowledge and future perspectives. Forest ecology and management 402: 102-114. Doi: 10.1016/j.foreco.2019.117678.

Vellinga, E. C., B. E. Wolfe and A. Pringle. 2009. Global patterns of ectomycorrhizal introductions. New Phytologist 181(4): 960-973. Doi: 10.1111/j.14698137.2008.02728.x.

VIk, L., L. Tedersoo, T. Antl, T. Větrovský, K. Abarenkov, J. Pergl, J. Albrechtová, M. Vosátka, P. Baldrian, P. Pysek and P. Kohout. 2020. Early successional ectomycorrhizal fungi are more likely to naturalize outside their native range than other ectomycorrhizal fungi. New Phytologist. Doi: 10.1111/nph.16557.

\section{(c) (i) (9)}

Todos los textos publicados por la Revista Mexicana de Ciencias Forestales -sin excepción- se distribuyen amparados bajo la licencia Creative Commons 4.0 Atribución-No Comercial (CC BY-NC 4.0 Internacional), que permite a terceros utilizar lo publicado siempre que mencionen la autoría del trabajo y a la primera publicación en esta revista. 